\title{
Molecular ordering of precursor films during spreading of tiny liquid droplets
}

\author{
M. Haataja ${ }^{1,2}$, J. A. Nieminen ${ }^{1}$, and T. Ala-Nissila ${ }^{1-3}$ \\ ${ }^{1}$ Department of Physics, Tampere University of Technology, \\ P.O. Box 692, FIN-33101 Tampere, Finland \\ ${ }^{2}$ Research Institute for Theoretical Physics, \\ P.O. Box 9 (Siltavuorenpenger 20 C), FIN-00014 University of Helsinki, Finland \\ ${ }^{3}$ Department of Physics, Brown University, Box 1843, Providence, R.I. 02912, U.S.A.
}

(21.7.1995)

In this work we address a novel feature of spreading dynamics of tiny liquid droplets on solid surfaces, namely the case where the ends of the molecules feel different interactions to the surface. We consider a simple model of dimers and short chain-like molecules which cannot form chemical bonds with the surface. We study the spreading dynamics by Molecular Dynamics techniques. In particular, we examine the microscopic structure of the time-dependent precursor film and find that in some cases it can exhibit a high degree of local order. This order persists even for flexible chains. Our results suggest the possibility of extracting information about molecular interactions from the structure of the precursor film.

68.10.Gw,61.20.Ja,05.70.Ln

Since the discovery of dynamical layering by Heslot et al. [1], there has been increasing interest in phenomena occuring at microscopic length-scales during spreading. The beautiful experiments of Refs. [1, 1 - reveal that both the molecular structure of the liquid and the type of substrate used influence density profiles of the droplets. For example, thickness profiles of tetrakis (2-ethylexoxy)silane and polydimethylsiloxane (PDMS) droplets on a silicon wafer exhibit strikingly different shapes under spreading [1]. Tetrakis exhibits clearly observable dynamical layering, while the spreading of PDMS proceeds by a fast evoluting precursor layer of one molecular thickness. Furthermore, the experiments of Heslot et al. [2] and Valignat et al. [5] also demonstrate the important role of liquid-surface interactions in determining the morphology of spreading droplets in microscopic scales.

Because of these experiments theoretical work has gained new interest. Analytic theories to date deal with dynamical layering only [6.7]. A number of computer simulations have been performed $[8$ 15] to study the dynamics of spreading with some emphasis on the molecular structure of the liquid. In particular, using Molecular Dynamics (MD) simulations it was concluded in Refs. 12] that the chain-like nature of the molecules can influence the structure of the precursor layer.

The molecular structure of thin layers is of practical importance, too. One way of controlling the surface energetics of a solid substrate is to use grafted molecules that adsorb on it, sometimes forming chemical bonds and brushed layers 16]. Such surfaces have applications in e.g. coating and lubrication. Another important class of systems comprises amphiphilic molecules such as detergents where a strong asymmetry of interactions causes layered structures to form 17]. Spreading dynamics of such molecules is then of particular interest in trying to understand how these layers form, and how well ordered they are.

Motivated by these considerations, we have chosen to study the dynamics of spreading of simple models of short, asymmetrically interacting molecules using MD simulations. This method is convenient since microscopic information about the structure and properties of the spreading layers is readily available in real time. Our model follows Ref. [12 where short chain-like molecules interact with each other via the Lennard-Jones (LJ) potential $V(r)=4 \epsilon_{f}\left(\left(\sigma_{f} / r\right)^{12}-\left(\sigma_{f} / r\right)^{6}\right)$, with parameters $\sigma_{f}$ and $\epsilon_{f}$ for the width and depth of the potential, respectively. The $n$-mers comprise $n$ LJ particles interconnected by a very rigid pair potential $V_{c}=(1 / 2) k\left(r-r_{0}\right)^{2}$, where $k=10000 \epsilon_{f} / \sigma_{f}^{2}$.

The surface is modelled by a flat, continuum LJ material with a pairwise LJ interaction integrated over the volume $z \leq 0$ to obtain an interaction potential $V_{i}(z)=-A / z^{3}+B / z^{9}$, where $A=(2 \pi / 3) \epsilon_{s} \sigma_{s}^{6} \rho$, and $B=(4 \pi / 45) \epsilon_{s} \sigma_{s}^{12} \rho$ [12], with $\rho \sim 10^{30} \mathrm{~m}^{-3}$. Table I shows the parameters. Interaction asymmetry comes about through the choice of the potentials: $V_{I}$ for one end of the chain (called the grafted end) and either $V_{I I}$ or $V_{I I I}$ for the other. The potential $V_{I}$ is much steeper and deeper than $V_{I I}$ and $V_{I I I}$, both of which are of equal depth. In addition, the equilibrium distance of the surface potential minimum as seen by the monomers is the same for $V_{I}$ and $V_{I I}$ (the ordinary case), but for $V_{I I I}$ (the shifted case) the minimum distance is about two bond lengths further away from the surface. For the shifted case this means that individual dimers tend to lie perpendicular to the surface.

The simulations are done at a constant temperature of $k T_{s}=0.8 \epsilon_{f}$, which is well above the triple point of an LJ material [12]. The dynamics is described by the equations of motion with a Nosé-Hoover thermostat 12, 18, 19. They are solved using a modified velocity Verlet algorithm [20]. The Hamaker constant $A_{H}$ is determined by the effective bond length $b_{l}$ of the dimers; e.g. for $b_{l} \approx 100 \AA, A_{H} \approx 10^{-18} \mathrm{~J}$ which is a realistic value for surfaces [17]. Assuming that the effective mass of the molecules is about $10^{5}$ amu [5], the time step in reduced 
units (r.u.) is $t_{r} \approx 10^{-13}$ seconds.

Fig. 1 shows snapshots of typical evolution of dimer droplets during spreading for the two cases. The random initial state of the ridge -shaped droplet is first carefully prepared, after which the surface interaction is turned on and spreading occurs perpendicular to the axis of the ridge. The dimers above the surface are mainly oriented perpendicular to it due to the stronger attraction of the grafted end. Dimers on the surface, however, behave quite differently. In the ordinary case the precursor film appears rather rough except very close to the edges of the droplet where the molecules have enough room to lie flat. In the shifted case the situation changes dramatically and the precursor film appears very well ordered at all stages of spreading.

To quantify these observations, we have followed the time evolution of various density-density autocorrelation functions 20. In particular, we have defined the precursor pair correlation function as follows:

$$
g\left(\left|\overrightarrow{r_{i}}-\overrightarrow{r_{j}}\right|, t\right)=N(t)^{-2}<\sum_{i} \sum_{j \neq i} \delta\left(\overrightarrow{r_{i}}\right) \delta\left(\overrightarrow{r_{j}}\right)>.
$$

The total number of molecules within the precursor film is denoted by $N(t), \overrightarrow{r_{i}}(t)$ is the position vector of the centre-of-mass of the $i^{\text {th }}$ dimer, and the summations go over the dimers in the precursor layer. This function has been computed during the spreading dynamics. After a short inital transient, it assumes a characteristic steady-state shape for the times studied here. For the ordinary case, $g$ has fairly sharp peaks corresponding to nearest neighbour dimers only which indicates that the layer is disordered and liquid-like. In the shifted case, however, clear and sharp peaks can be observed corresponding up to about fourth or fifth nearest neigbour dimers. The precursor layer in this case has a high degree of local order even at these elevated temperatures. We have also followed the evolution of an orientational distribution function which provides us with information about the relative orientations of neighbouring dimers. For the shifted case a sharp peak is observed corresponding to parallel orientations, which indicates that the orientations of neighbouring dimers are strongly correlated, too. In both cases the very late time regime corresponds to a diffusively thinning monolayer.

Fig. 2 shows typical smoothed density profiles of dimers at three different times. In the ordinary case the profiles are fairly smooth and rounded, and no layering is present. At later times a step develops at the edges of the film where dimers tend to lie flat on the surface. In the shifted case the edge of the precursor film always remains very sharp and well-defined. It is conceivable that tendency towards layering on top of the precursor layer might be observed for larger droplets in this case.

We have also studied the time-dependence of the precursor film width $w(t)$. Fig. 3 shows a comparison between the two cases. They are very similar, with a more rapid "almost linear" $\left(\sim t^{0.9}\right)$ region followed by a crossover to slower "diffusive" $\left(\sim t^{0.5}\right)$ behaviour. This is characteristic of the truly microscopic droplets studied here and has been observed in Refs. 10,12 as well. For our cylindrical geometry, within the first region $w(t) \propto N(t) \propto t^{0.9}$, which for a true $3-d$ droplet means that its radius $R(t) \sim \sqrt{N} \sim t^{0.45}$ since the flux into the first layer remains roughly constant. This overall $R(t) \sim t^{0.5}$ behaviour is in agreement with experiments [20.

Furthermore, it is possible to estimate the corresponding effective diffusion coefficients in the two regimes. In the ordinary case for the "truly" diffusive late-time regime we find $D_{\ell} \sim 10^{-6} \mathrm{~m}^{2} / \mathrm{s}$ [21]. For the shifted case $D_{\ell}$ is about an order of magnitude smaller because the activation energy arising from neighbouring dimers is much larger due to the close-packed geometry of the layer. Most importantly, however, estimating a "diffusion" coefficient $D_{e}$ from the early-time (almost) linear region, we find that $D_{e} / D_{\ell} \approx 100$. This is in surprisingly good quantitative agreement with recent experiments, where the same ratio was measured between early-time and late-time "diffusion" coefficients [5.

Additional studies of flexible chains consisting of four and eight particles where one of the ends is grafted with $V_{I}$ reveal that the spreading becomes similar to the results of Refs. [12] without any asymmetry of interaction, provided that the equilibrium orientation of an individual chain is parallel to the surface. In the case of a shifted potential, however, in which the equilibrium orientation of an individual chain is perpendicular to the surface, compact and well-ordered structure of the precursor layer still persists even for completely flexible chains. Complete results with different shifted potentials will be published elsewhere.

Due to the microscopic nature of our droplets, it is difficult to make comparisons with experiments. A spreading experiment with grafted polymer chains has been performed by Valignat et al. [5]. In some cases, the molecules become anchored on the surface and spreading stops. However, some of their density profiles do qualitatively resemble ours. For example, in the case of a microdroplet of PDMS-OH on a silicon wafer covered with a layer of behenic acid, a well-defined and sharp precursor film develops similar to our shifted case. We are not aware of any experiments probing microscopic order within the precursor film. However, it is interesting to note that in the experiments of Ref. [22], equilibrium ordering of thin layers of a simple liquid between two corrugated surfaces was observed.

In conclusion, we have shown further evidence of how the surface-chain interactions can play a significant role in determining the microscopic structure of the precursor layer. In particular, through a simple model we have demonstrated how the internal structure of the film can depend on the asymmetry of surface interactions between the chain ends. This can lead to a high degree of local order if the molecules prefer a vertical equilibrium orienta- 
tion. Additionally, our model reproduces the experimentally observed more rapid spreading at the early stages, which crosses over to slower diffusion at late times. Based on our results we suggest the possibility of obtaining information about the nature of these interactions by monitoring the density profile and precursor film during spreading.

[1] F. Heslot, N. Fraysse, and A. M. Cazabat, Nature 338, 640 (1989).

[2] F. Heslot, A. M. Cazabat, and P. Levinson, Phys. Rev. Lett. 62, 1286 (1989).

[3] F. Heslot, A. M. Cazabat, P. Levinson, and N. Fraysse, Phys. Rev. Lett. 65, 599 (1990).

[4] U. Albrecht, A. Otto, and P. Leiderer, Phys. Rev. Lett. 68, 3192 (1992).

[5] M. P. Valignat, N. Fraysse, A. M. Cazabat, and F. Heslot, Langmuir 9, 601 (1993).

[6] D. B. Abraham, P. Collet, J. De Coninck, and F. Dunlop, Phys. Rev. Lett. 65, 195 (1990).

[7] P.-G. de Gennes and A.-M. Cazabat, C. R. Acad. Sci. Paris Ser. II 310, 1601 (1990).

[8] J. Heiniö, K. Kaski, and D. B. Abraham, Physica Scr. T38, 28 (1991).

[9] J.-x. Yang, J. Koplik, and J. R. Banavar, Phys. Rev. Lett. 67, 3539 (1991); Phys. Rev. A 46, 7738 (1992).

[10] J. A. Nieminen, D. B. Abraham, M. Karttunen, and K. Kaski, Phys. Rev. Lett. 69, 124 (1992).

[11] J. De Coninck, N. Fraysse, M. P. Valignat, and A. M. Cazabat, Langmuir 9, 1906 (1993).

[12] J. A. Nieminen and T. Ala-Nissila, Europhys. Lett. 25, 593 (1994); Phys. Rev. E 49, 4228 (1994).

[13] O. Venäläinen, T. Ala-Nissila, and K. Kaski, Europhys. Lett. 25, 125 (1994); Physica A 211, 214 (1994).

[14] L. Wagner, Phys. Rev. E 51, 499 (1995).

[15] J. De Coninck, U. D'Ortona, and J. R. Banavar, Phys. Rev. Lett. 74, 928 (1995).

[16] S. T. Milner, Science 251, 905 (1991).

[17] J. Israelachvili, Intermolecular \& Surface Forces, 2nd ed., Academic Press, London, U.K. (1992).

[18] S. Nosé, J. Chem. Phys. 81, 511 (1984).

[19] W. G. Hoover, Phys. Rev. A 31, 1695 (1985).

[20] M. P. Allen and D. J. Tildesley, Computer Simulation of Liquids, Oxford University Press, Oxford, U.K. (1987).

[21] This is somewhat larger than the measured values (see e.g. Refs. [2.3.5]) which is not surprising since in our units $T \approx 1600 K$, and there are no surface diffusion barriers.

[22] J. N. Israelachvili, P. M. McGuiggan, and A. M. Homola, Science 240, 189 (1988). 


\begin{tabular}{||c|c|c||}
\hline \hline$\epsilon_{s}$ & $\sigma_{s}$ & Potential \\
\hline $1.0 \epsilon_{f}$ & $5.0 \sigma_{f}$ & $V_{I}$ \\
\hline $0.06 \epsilon_{f}$ & $5.0 \sigma_{f}$ & $V_{I I}$ \\
\hline $0.02 \epsilon_{f}$ & $7.3 \sigma_{f}$ & $V_{I I I}$ \\
\hline \hline
\end{tabular}

TABLE I. Surface-monomer interaction parameters for the two cases discussed in the text. 


\section{FIGURE CAPTIONS}

Fig. 1. Snapshots of a dimer droplet for the ordinary and the shifted cases. The grafted end is represented by a filled circle. (a) Initial configuration for both cases (as seen along the axis of the ridge). (b) The ordinary case at $t=30000$ r.u., and (c) the shifted case at $t=30000$ r.u. . Differences in the morphology of the precursor layer are clearly visible.

Fig. 2. Smoothed density profiles for the two cases: (a) the ordinary case, and (b) the shifted case. The shoulders at the edge of the precursor film in (a) are due to dimers that fall flat on the surface.

Fig. 3. Width of the precursor film $w(t)$ vs. time for the two cases discussed in the text. This data is for 1525 dimers. Note the considerably slower late-time behaviour of the shifted case. 